$\overline{A E E T}$

ASOCIACIÓN ESPAÑOLA DE ECOLOGÍA TERRESTRE

\title{
Las huellas de la defaunación en el Antropoceno: el colapso de los mutualismos de dispersión de semillas
}

\author{
N. Pérez-Méndez ${ }^{1}$ \\ (1) Departamento de Ecología Integrativa, Estación Biológica de Doñana (EBD-CSIC), C/Americo Vespucio s/n, La Cartuja, 41092 Sevilla, España. \\ * Autor de correspondencia: N. Pérez-Méndez [nestorperezmendez@gmail.com]
}

> Recibido el 27 de julio de 2016 - Aceptado el 23 de septiembre de 2016

Pérez-Méndez, N. 2016. Las huellas de la defaunación en el Antropoceno: el colapso de los mutualismos de dispersión de semillas. Ecosistemas 25(3):134-137. Doi.: 10.7818/ECOS.2016.25-3.17

El ritmo de crecimiento de la población humana mundial en los últimos 500 años ha sido vertiginoso, pasando de menos de 500 millones de habitantes a más de 7000 millones en la actualidad. Paralelamente, el ritmo de pérdida de biodiversidad asociado a este crecimiento, ha aumentado hasta niveles sólo comparables con los cinco grandes eventos de extinción masiva de la historia de la Tierra. A pesar de que este fenómeno ocurre en todos los grupos taxonómicos, hay ciertos rasgos, como un tamaño corporal grande, que hacen a algunas especies más vulnerables que a otras. A menudo estas especies cumplen roles funcionales muy importantes y poco redundantes, por lo que las consecuencias de su pérdida pueden ser críticas para el funcionamiento de los ecosistemas. Por un lado, por la pérdida directa de las funciones que desempeñan $y$, por otro, porque a menudo los efectos se propagan en cascada afectando a la estructura, la composición y la dinámica de las comunidades alteradas. Es importante, por tanto, desarrollar nuevas vías de investigación que permitan entender y predecir cuáles son los efectos en cascada y las consecuencias potenciales de la defaunación mediada por la actividad antrópica y de la pérdida de funciones ecológicas asociadas.

La dispersión de semillas es un proceso clave en el ciclo de regeneración natural de las plantas, que en gran medida determina las características demográficas y genéticas de sus poblaciones. Muchas especies de plantas dependen para su dispersión de semillas de relaciones mutualistas con animales frugívoros, que reciben a cambio recursos tróficos. Sin embargo, la alteración antrópica del medio ha provocado la extinción de muchos de estos animales, especialmente los de mayor tamaño, provocando una reducción de tamaño en las comunidades de frugívoros. Estas especies de mayor tamaño normalmente ofrecen unos mejores servicios de dispersión a las plantas. Por un lado, consumen un mayor número de frutos y de mayor tamaño $y$, por otro, suelen dispersar las semillas a mayores distancias. A largo plazo sería esperable, por tanto, que la reducción de tamaño progresiva de los frugívoros provocara una disminución de los servicios de dispersión con consecuencias genéticas y demográficas para las plantas.
El objetivo general de esta tesis doctoral fue evaluar las consecuencias de la degradación progresiva y del colapso de los servicios de dispersión asociados a una pérdida gradual de tamaño de los dispersores de semillas en distintos aspectos funcionales (demográficos y genéticos) de las poblaciones de plantas endozoócoras. Para responder estas cuestiones se estudió la interacción mutualista entre la planta Neochamaelea pulverulenta (Rutaceae) y sus únicos dispersores de semillas, los lagartos frugívoros del género Gallotia (Lacertidae). N. pulverulenta es un arbusto endémico de las Islas Canarias que produce frutos carnosos (drupáceos) con semillas de gran tamaño. Éstos son consumidos exclusivamente por lagartos medianos y grandes del género Gallotia, también endémico del archipiélago. Hasta la llegada de los humanos a las islas, hace aproximadamente 2500 años, los lagartos gigantes de este género eran abundantes en cada una de las tres islas donde se distribuye N. pulverulenta (Gran Canaria, Tenerife y La Gomera), con individuos que podían superar los $500 \mathrm{~mm}$ de longitud hocico-cloaca (LHC). Sin embargo, como consecuencia directa o indirecta de la actividad humana sólo en Gran Canaria se preservan individuos de tamaño relativamente grande en la actualidad ( $G$. stehlini; LHC max $=280 \mathrm{~mm}$ ), mientras que en Tenerife sólo son abundantes individuos de tamaño mediano (G. galloti, LHC max= $145 \mathrm{~mm}$ ) y en La Gomera individuos pequeños $(G$. caesaris; LHC $\max =111 \mathrm{~mm}$ ). A través de una aproximación comparativa entre estos escenarios contrastados desde el punto de vista del tamaño de los dispersores de semillas (Fig. 1) se analizaron los efectos demográficos y genéticos en las poblaciones de N. pulverulenta.

\section{Efectos demográficos de la defaunación}

La degradación del proceso de dispersión de semillas asociado a la pérdida de tamaño de los dispersores de semillas debería ocasionar impactos negativos en la dinámica demográfica en las poblaciones plantas. Los resultados obtenidos en esta tesis, demuestran que, efectivamente, la regeneración de $N$. pulverulenta 


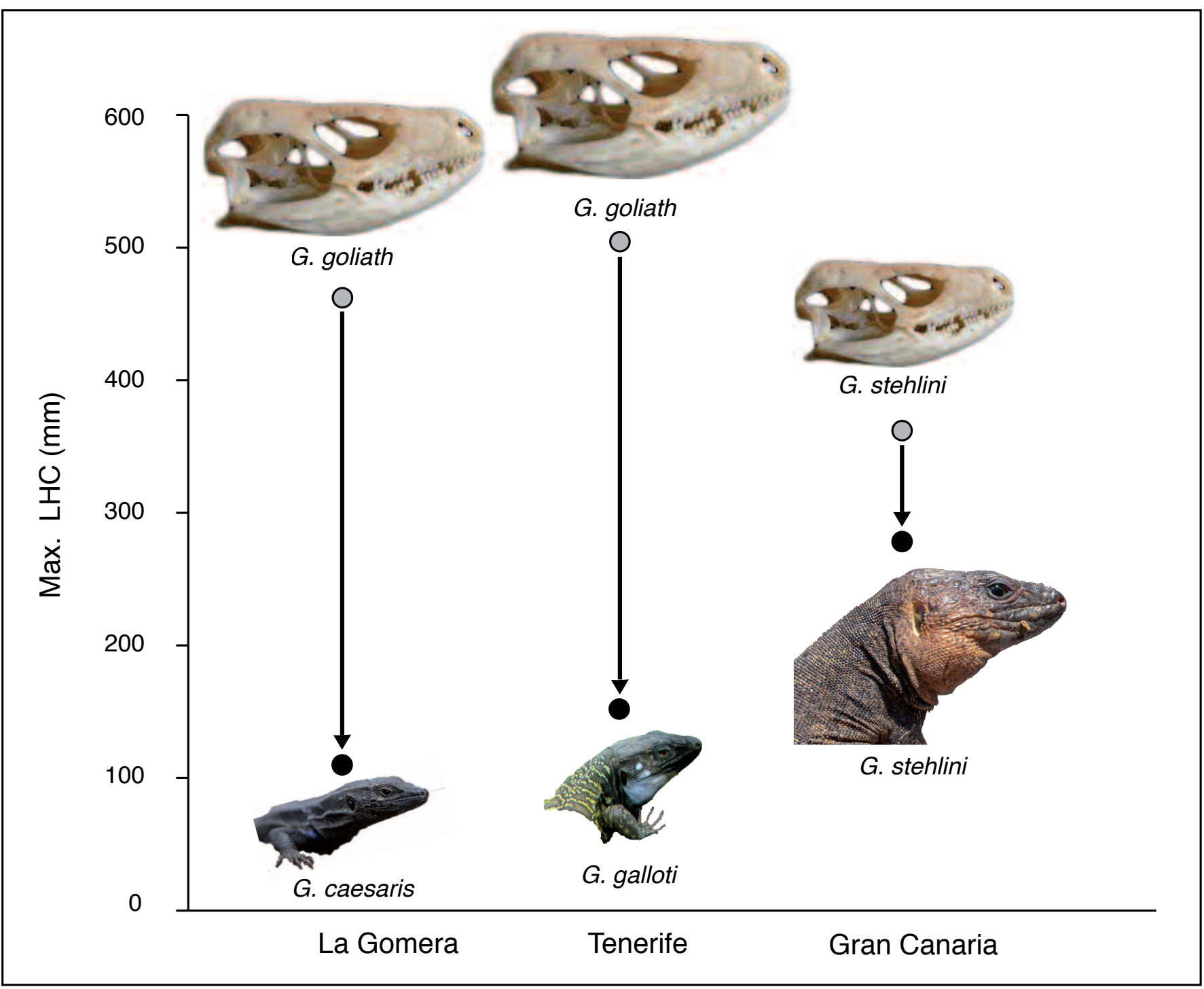

Fig.1 Esquema de la reducción de tamaño (Iongitud hocico-cloaca máxima, Max. LHC) de los lagartos frugívoros de las Islas Canarias (Gallotia sp.) como consecuencia del proceso de defaunación iniciado con el asentamiento humano en las islas ( 2.500 años). En esta figura sólo se muestra la reducción de talla para las tres islas donde se distribuye Neochamaelea pulverulenta (Rutaceae). Fotos: G. caesaris (B. Rodríguez); G. galloti (C. Camacho); G. stehlini (A. Valido).

Fig.1 Scheme of body-size reduction (Maximum Snout to Vent Length; Max. LHC) of frugivorous lizards from the Canary Islands (Gallotia sp.) caused by a defaunation process following the arrival of humans to the islands ( 2500 years b.p.). This figure shows only body-size reduction in those islands where Neochamaelea pulverulenta (Rutaceae) is present. Photos: G. caesaris (B. Rodríguez); G. galloti (C. Camacho); G. stehlini (A. Valido).

se ve fuertemente afectada en los escenarios que sólo albergan lagartos de tamaño mediano (Tenerife) y pequeño (La Gomera) (Pérez-Méndez et al. 2015). Sin embargo, estos efectos sólo se evidencian cuando analizamos conjuntamente las componentes cuantitativa y cualitativa de la efectividad de la dispersión de semillas. Los datos muestran una estructura de edad similar en las tres islas, con un número similar de plántulas establecidas. Sin embargo, la tasa de reclutamiento efectiva, es decir la cantidad de plántulas que se establecen fuera del vecindario materno, donde la mortalidad suele ser menor, fue extremadamente baja en la isla más defaunada (La Gomera). Esto ocurre porque G. caesaris, el único lagarto abundante en esta isla tras la extinción de los lagartos gigantes, no presenta tamaño lo suficientemente grande como para consumir frutos de $N$. pulverulenta. Inesperadamente, la tasa de reclutamiento efectivo en Tenerife fue significativamente mayor que en Gran Canaria, donde se conservan los lagartos de mayor tamaño. Esto puede ser explicado por una mayor abundancia (seis veces mayor) de lagartos medianos (G. galloti) en Tenerife que de lagartos grandes (G. stehlini) en Gran Canaria. Sin embargo, el vigor de las plántulas establecidas en Tenerife es mucho menor que en Gran Canaria, como consecuencia de la dispersión de un rango de semillas más pequeñas.

\section{Efectos genéticos de la defaunación}

La cantidad de variación genética de las plantas y, su distribución espacial, viene determinada por la acción conjunta del flujo de genes dentro y entre poblaciones, así como otros procesos como la selección, la deriva génica y la tasa de mutación. Por tanto, dado que los animales de mayor tamaño tienden a moverse mayores distancias, la reducción de talla de los vertebrados frugívoros debería ocasionar una serie de efectos en cascada, con una disminución del flujo de genes vía semillas y una alteración de las características genéticas de las poblaciones de plantas afectadas. Efectivamente, los resultados obtenidos en esta tesis muestran una reducción drástica de las distancias de dispersión de semillas que refleja el gradiente de pérdida de tamaño de los lagartos (Fig. 2). En Gran Canaria se detectan distancias de dispersión mucho mayores (distancia máxima= $94 \mathrm{~m}$ ) que, en Tenerife, donde existe muy pocos eventos de dispersión a larga 


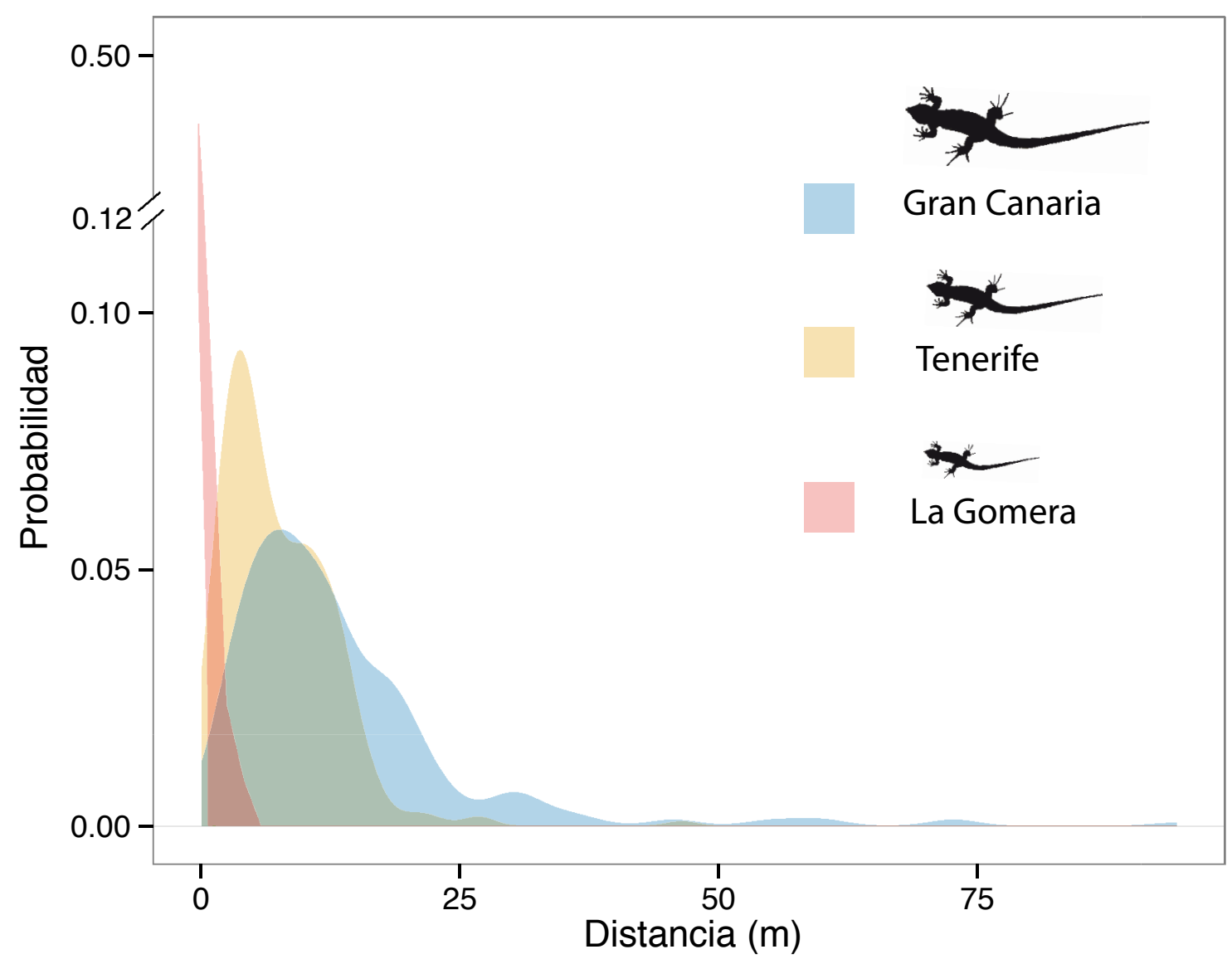

Fig.2. Distribución de frecuencias de las distancias de dispersión de semillas de Neochamaelea pulverulenta en los tres escenarios ecológicos analizados. Las áreas sombreadas representan la curva de probabilidad estimada a partir de la distribución empírica de distancias de dispersión (Pérez-Méndez et al. 2016), evaluada con técnicas genéticas basadas en genotipado del endocarpio de las semillas y su comparación con el de plantas adultas.

Fig. 2 Frequency distribution of seed dispersal distances of Neochamaelea pulverulenta in the three ecological scenarios. Shaded areas indicate the probability curves estimated from the empirical distribution of dispersal distances (Pérez-Méndez et al. 2016). Empirical dispersal distances were determined with genetic tools based on the genotyping of seed endocarps and seed sources (i.e. adult plants).

distancia (distancia máxima= $46 \mathrm{~m}$ ) y una sobre representación de eventos a corta distancia. En La Gomera prácticamente todas las semillas se encuentran debajo de la planta madre, excepto una mínima proporción que se dispersa, de forma esporádica, fuera del vecindario materno más próximo (distancia máxima= $4 \mathrm{~m}$ ) (PérezMéndez et al. 2016).

Esta reducción del flujo de genes vía semillas tiene un impacto muy marcado en la distribución de la variación genética a distintas escalas espaciales. A escala local, a medida que las distancias de dispersión de semillas de $N$. pulverulenta se acortan, se reduce el solapamiento de la sombra de semillas de los individuos reproductivos. Se generan de esta manera vecindarios de individuos muy emparentados genéticamente. El resultado es un incremento del grado de estructuración genética espacial que refleja el gradiente de pérdida de tamaño de los lagartos frugívoros en los tres escenarios (La Gomera > Tenerife > Gran Canaria) (Pérez-Méndez et al. 2016).

Comparando la diferenciación genética entre poblaciones a escala insular, por medio de redes que representan su conectividad genética a amplia escala geográfica, encontramos evidencias de que la reducción del flujo génico vía semillas se extiende más allá de la escala local (Fig. 3). Mientras que la red de Gran Canaria preserva una mayor conectividad genética entre poblaciones, en Tenerife se observan signos de una ligera reducción de la misma. Este efecto es mucho más marcado cuando la dispersión de semillas colapsa, por extinción de los grandes lagartos, en el escenario de La Gomera, donde la conectividad genética entre poblaciones desciende bruscamente para mantenerse sólo hasta unos pocos kilómetros $(\sim 4 \mathrm{~km})$. Consistentemente, el aislamiento genético por distancia en esta isla fue mucho más acentuado que en los otros dos escenarios (Pérez-Méndez et al. 2016).

\section{Conclusiones}

Los resultados de esta tesis doctoral revelan los múltiples efectos en cadena asociados a la extinción de los grandes animales frugívoros y de las interacciones ecológicas en las que participan. En este caso, una degradación progresiva de los servicios de dispersión de semillas, que deriva, a su vez, en la alteración de la dinámica demográfica y las características genéticas de las plantas con las que interaccionan. Se pone de manifiesto así la necesidad de conservar a los vertebrados de mayor tamaño para evitar no sólo la pérdida de estas especies, sino de todo el rango de procesos en los que intervienen. De otra forma, las consecuencias de la defaunación en el funcionamiento de los ecosistemas pueden aparecer mucho después de la extinción de estas especies, a veces de formas tan poco evidentes como las que se evidencian en esta tesis doctoral (Pérez-Méndez 2016).

\section{Agradecimientos}

A mis directores de tesis, Alfredo Valido y Pedro Jordano, al Grupo de Ecología Integrativa de la Estación Biológica de Doñana y a todo aquel que, de una manera $u$ otra, ha contribuido a que esta tesis sea una realidad. Esta tesis fue financiada por el Ministerio de Economía y Competitividad (MINECO) a través del proyecto de investigación CGL2009-09715, que incluyó una beca pre-doctoral FPI (BES-2010-041463). 


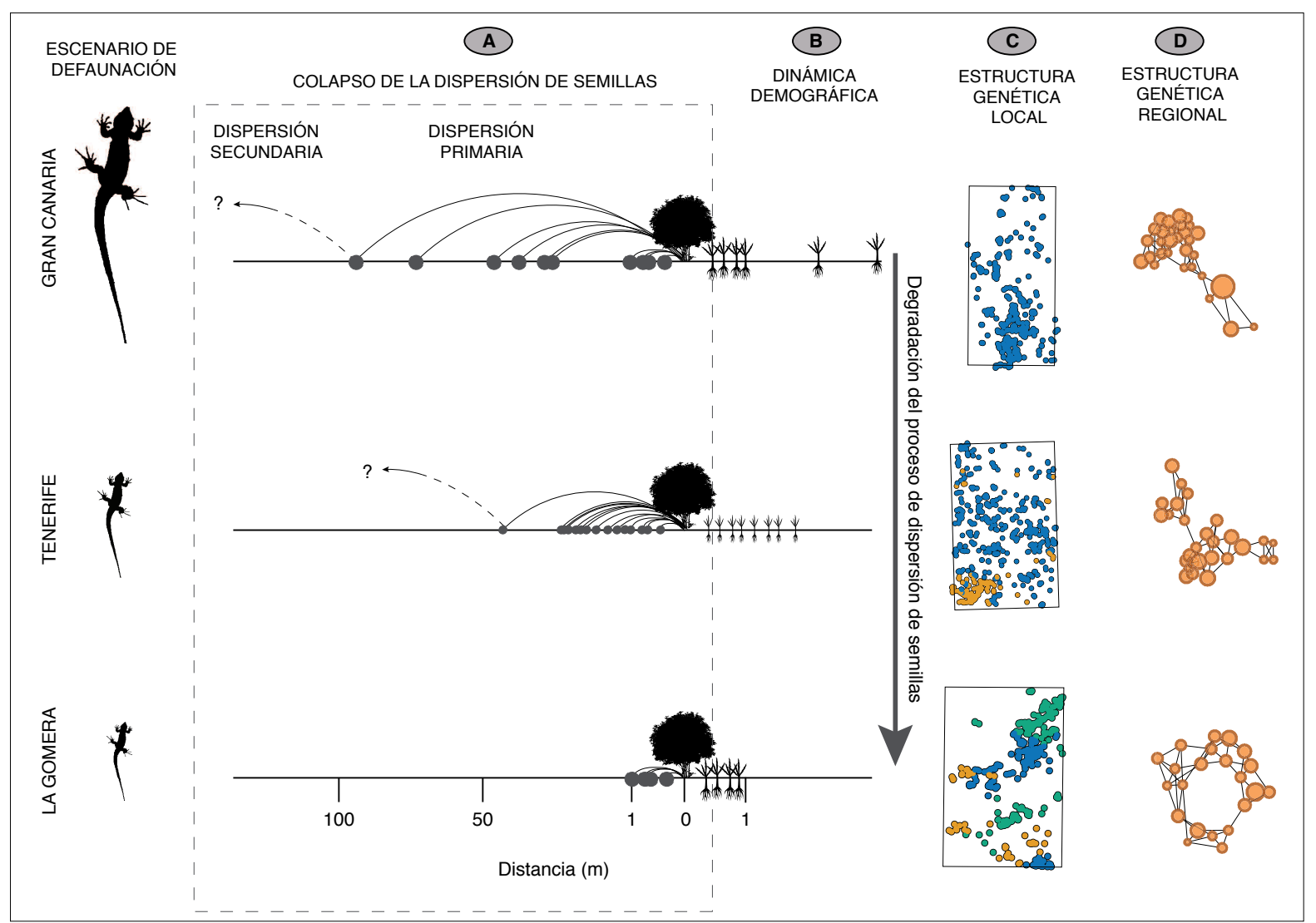

Fig. 3 Resumen de los efectos en cascada de la reducción de talla de los lagartos frugívoros (Gallotia spp.) en tres escenarios insulares de defaunación. A: Degradación progresiva, y eventual colapso, del proceso de dispersión de semillas de Neochamaelea pulverulenta (Rutaceae), en términos de distancias de dispersión. B: Consecuencias demográficas de la defaunación en términos de reclutamiento efectivo (>1 m de planta adulta) y vigor de plántulas establecidas. C: Consecuencias genéticas a escala local (dentro de poblaciones) en términos de generación de vecindarios genéticos (clusters genéticos) de mayor parentesco entre plantas individuales. Cada círculo corresponde a un individuo adulto de las poblaciones analizadas ( 1 ha) y cada color indica a qué cluster genético ( $k$ ) corresponden. El número de clusters aumenta desde Gran Canaria ( $k=1)$, Tenerife ( $k=2)$ hasta La Gomera ( $k=3)$. D: Consecuencias genéticas a escala regional (entre poblaciones dentro de isla) en términos de conectividad genética. Cada nodo de la red representa una población muestreada a escala insular. Cada enlace indica que existe conectividad genética entre dos poblaciones y la longitud del enlace es proporcional a la distancia genética condicional entre poblaciones, por tanto, se infiere mayor conectividad con enlaces más cortos. La topología de la red de conectividad genética de Gran Canaria es considerablemente más compacta (más enlaces por nodo y enlaces más cortos) que la red de La Gomera. Ello indica pérdidas de conectividad a amplia escala geográfica en el escenario más defaunado, donde el proceso de dispersión de semillas no es funcional. Más detalles en las correspondientes publicaciones.

Fig. 3 Summary of cascading effects triggered by body-size reduction of frugivorous lizards (Gallotia sp.) in the three scenarios of defaunation. A: Progressive degradation and eventual collapse of the seed dispersal process of Neochamaelea pulverulenta (Rutaceae) in terms of dispersal distances. B: Demographic consequences of defaunation in terms of effective recruitment ( $>1 \mathrm{~m}$ from adult plants) and vigour of established seedlings. C: Genetic consequences at local scale (within populations) with local plant neighborhoods showing higher genetic similarity when large-sized dispersers become extinct. Circles represent individual plants within sampled populations $(1 \mathrm{Ha})$ and colours indicate different genetic clusters $(\mathrm{k})$. The number of clusters increases from Gran Canaria ( $k=1)$, to Tenerife ( $k=2$ ), and La Gomera ( $k=3)$. D: Genetic consequences at regional scale (among populations) in terms of genetic connectivity among populations. Nodes represent sampled populations within the three islands. Links indicate genetic connectivity among pairs of populations and link length is proportional to the conditional genetic distance among them (i.e. the shorter the link, the higher the connectivity among populations). Network topology in Gran Canaria is more compact (a larger number of links per node and shorter links) than that of La Gomera, suggesting a loss of genetic connectivity in the most defaunated scenario, where the seed dispersal process is not functional.

\section{Referencias}

Pérez-Méndez, N., Jordano, P., Valido, A. 2015. Downsized mutualisms: consequences of seed dispersers' body-size reduction for early plant recruitment. Perspectives in Plant Ecology, Evolution and Systematics 17: 151-159.
Pérez-Méndez, N. 2016. Las huellas de la defaunación en el Antropoceno. El colapso de los mutualismos de dispersión de semillas. Universidad Pablo de Olavide. Sevilla. España.

Pérez-Méndez, N., Jordano, P., García, C., Valido, A. 2016. The signatures of Anthropocene defaunation: cascading effects of the seed dispersal collapse. Scientific Reports 6: 24820.

\section{NÉSTOR PÉREZ-MÉNDEZ}

Las huellas de la defaunación en el Antropoceno: el colapso de los mutualismos de dispersión de semillas

Tesis Doctoral

Departamento de Ecología Integrativa. Estación Biológica de Doñana (EBD-CSIC)

Julio 2016

Directores: Alfredo Valido y Pedro Jordano 